\title{
AGRUPAMENTO DE FAMÍLIAS DE MEIO-IRMÃOS DE Pinus taeda BASEADO EM CARACTERÍSTICAS FÍSICAS E MECÂNICAS DA MADEIRA
}

\author{
${ }^{1}$ Sílvia Aparecida Angelo Romão; ${ }^{2}$ Jorge Luis Monteiro de Matos; ${ }^{3}$ Luciana Duque Silva; \\ ${ }^{4}$ Antonio Rioyei Higa \\ ${ }^{1}$ Eng ${ }^{\mathrm{a}}$ Industrial Madeireira, M.Sc., Curitiba, PR, Brasil - silvia_romao@yahoo.com.br \\ ${ }^{2}$ Eng. Florestal, Dr., Depto. de Engenharia e Tecnologia Florestal, Curitiba, PR, Brasil - jmatos.ufpr@gmail.com \\ ${ }^{3}$ Eng $^{\mathrm{a}}$ Florestal, Dr ${ }^{\mathrm{a}}$., USP/ESALQ, Piracicaba, SP, Brasil - lucianaduques@usp.br \\ ${ }^{4}$ Eng. Florestal, Dr., Depto. de Ciências Florestais, UFPR, Curitiba, PR, Brasil - antonio.higa@gmail.com
}

Recebido para publicação: 22/09/2010- Aceito para publicação: 14/08/2012

\begin{abstract}
Resumo
Este trabalho teve como objetivo agrupar famílias de um teste de progênies de Pinus taeda L. com base nas propriedades físicas e mecânicas da madeira, para inclusão dessas características qualitativas da madeira em um programa de melhoramento genético. As variáveis analisadas foram: massa específica básica e aparente, coeficientes de contração máxima volumétrica e linear tangencial, radial e longitudinal, anisotropia de contração e coeficientes de retratibilidade volumétrico, tangencial e radial, MOE, MOR e tensão de cisalhamento. As árvores avaliadas pertenciam a um teste de progênies instalado em blocos casualizados com cinco repetições, 120 famílias. Dentre as cinco plantas por parcela foi amostrada uma. Foram coletados os discos base, 25\%, 50\%, 75\% e 100\% da altura, para determinação da massa específica básica. A primeira e segunda toras foram utilizadas na preparação de amostras para as demais análises físicas e mecânicas. Na análise do experimento, foi utilizada a análise de componentes principais e de agrupamento. A análise de componentes principais permitiu destacar seis autovalores, explicando $82,51 \%$ da variância total das observações, e a análise de agrupamento possibilitou o agrupamento das 120 famílias em seis grupos, os quais apresentam distintas características de qualidade da madeira relacionadas à produção para diferentes finalidades. Palavras-chave: Análise multivariada; qualidade da madeira; aplicação tecnológica.
\end{abstract}

\begin{abstract}
Gathering of Pinus taeda families based on physical and mechanical wood characteristics. This research aimed to gather families of a Pinus taeda L. progeny test based on physical and mechanical properties of wood, in order to include these wood characteristics in a breeding program. The variables focused were: specific gravity and apparent maximum volumetric shrinkage coefficients and linear tangential, radial and longitudinal shrinkage anisotropy coefficients and volumetric shrinkage, the tangential and radial MOE and MOR and shear stress. Trees evaluated were sampled (one plant per plot) in a progeny test planted in randomized blocks with 120 families, five replicates, and five plants per linear plot. After measuring the DBH on standing trees the discs were collected to evaluate the specific gravity, on the following positions: base, $25 \%, 50 \%, 75 \%$ and $100 \%$ of the commercial height. The first and second logs were used for sample preparation for other physical and mechanical analysis. The principal component analysis allowed to highlight six eigenvalues which explained $82.51 \%$ of the total observations variance, and cluster analysis allowed 120 families gathering in six groups, which have different quality characteristics related to production of wood for different purposes.

Keywords: Multivariate analysis; wood quality; technology application.
\end{abstract}

\section{INTRODUÇÃO}

De acordo com a Sociedade Brasileira de Silvicultura (SBS, 2008), o setor florestal representava $3,4 \%$ do PIB (produto interno bruto) nacional. Apesar dessa participação significativa na economia nacional, a contribuição do setor florestal poderá ser ainda melhorada, através de esforços conjuntos envolvendo governo, instituições de pesquisa e iniciativa privada, no desenvolvimento de tecnologias que 
proporcionem melhoria na qualidade das matérias-primas usadas na fabricação de produtos de origem florestal, tanto de florestas nativas como de plantações florestais manejadas adequadamente.

Atualmente, a produção de madeira em plantações de ciclos curtos, com espécies de rápido crescimento, vem substituindo o suprimento de madeira de árvores adultas com grandes diâmetros, provenientes de florestas naturais. Apesar de a área anual de plantio ter sofrido uma queda em 2009, em consequência da crise internacional de 2008, a taxa de plantio está retornando aos patamares anteriores a 2008 (ABRAF, 2011). O governo brasileiro tem indicado essa direção. De acordo com o Plano Nacional de Mudanças Climáticas, lançado em 2008, por exemplo, a área com plantações florestais deverá atingir 11 milhões de ha em 2020 (SFB, 2010).

Em 2010, as plantações florestais ocupavam uma área de 6,5 milhões de ha, dos quais $27 \%$ eram com espécies do gênero Pinus sp. (ABRAF, 2011). A escala de produtos industriais madeiráveis a partir dessas florestas inclui, em geral, madeira serrada, madeira compensada e laminada, painéis de madeira reconstituídos (MDF, OSB, aglomerado etc.), componentes modulares (produtos laminados, molduras, pisos e revestimentos etc.), polpa e papel e cada vez mais a bioenergia (CARLE; HOLMGREN, 2008).

Com a crescente substituição da madeira de espécies nativas tradicionais pela madeira originada de plantações de pínus nas regiões Sul e Sudeste do Brasil, observa-se uma exigência cada vez maior na qualidade dessa madeira, necessitando ser mais homogênea e com a menor quantidade possível de nós e de outros defeitos (FOELKEL, 2008).

A maioria dos programas de melhoramento genético com espécies do gênero Pinus sp. é conduzida no Brasil por empresas de celulose e painéis do tipo MDF. Dessa forma, esses programas visam o aumento da produtividade da biomassa seca, que melhora o rendimento industrial, e melhoria na homogeneidade e retidão do fuste, que melhora o rendimento da colheita e transporte. Apesar do baixo rendimento de madeira sólida limpa no processamento mecânico, poucas iniciativas foram tomadas no sentido de incorporar as propriedades físicas e mecânicas da madeira nos programas de melhoramento genético dessa matéria-prima. Essa situação é resultante das dificuldades de se analisarem essas propriedades usando amostras de madeira representativas das árvores, coletadas de forma não destrutiva, e da impossibilidade de brotação da árvore adulta, para resgate do germoplasma e inclusão das árvores selecionadas na População Selecionada, para posterior cruzamentos e geração de novos indivíduos melhorados geneticamente.

Em geral, as variações verificadas nos diferentes materiais genéticos ocorrem de forma diferenciada, fazendo com que dificilmente se consiga encontrar grupos de árvores dotados de um número elevado de características desejadas (ASSIS, 2001). Assim considera-se importante a junção ou congregação dessas características em grupos de árvores, possibilitando a seleção de árvores que tenham maior quantidade de atributos tecnológicos desejáveis na sua madeira.

Alguns autores vêm investigando as propriedades da madeira por meio de técnicas que possibilitem a avaliação de um conjunto de características simultaneamente, para produção de celulose e carvão vegetal. Estudos nesse sentido foram realizados por Caixeta et al. (2003), Lopes (2003) e Rodrigues et al. (2008), que realizaram em seus trabalhos a seleção e classificação de genótipos superiores de Eucalyptus sp. baseados em índices de qualidade da madeira.

Diante do exposto, o presente estudo teve como objetivo geral agrupar famílias de um teste de progênies de Pinus taeda L. com base nas propriedades físicas e mecânicas da madeira, para inclusão dessas características qualitativas da madeira em um programa de melhoramento genético da espécie.

\section{MATERIAL E MÉTODOS}

\section{Descrição da área experimental e material genético}

O teste de progênies de Pinus taeda L., onde o estudo foi realizado, localiza-se no município de Rio Negrinho, região norte de Santa Catarina. A altitude local é de 985 m, a latitude de $26^{\circ} 40^{\prime} 07,24^{\prime \prime} \mathrm{S}$ e a longitude de 49 37’37,38” O. A média pluviométrica anual está entre 1500 e $1800 \mathrm{~mm}$, com umidade relativa média anual de 80 a $85 \%$.

O clima da região, segundo Köppen, é classificado como $\mathrm{Cfw}$ e pertence ao grupo $\mathrm{C}$ mesotérmico, com ocorrência de geadas, e com temperatura média mensal não atingindo mais que $22{ }^{\circ} \mathrm{C}$. O solo é do tipo Cambissolo Háplico, com base no levantamento e mapeamento de solo realizado na área.

O teste de progênies foi instalado em blocos casualizados, com 120 famílias de meio-irmãos, em parcelas lineares de cinco plantas com cinco repetições, plantadas em espaçamento $2,5 \mathrm{~m}$ x 2,5 m, com bordadura dupla. 
Para a amostragem das árvores, foi realizada a análise genética da variável de crescimento (DAP) aos 10 anos de idade, quando os indivíduos de cada parcela foram classificados de 1 a 5 , pelo respectivo valor genético individual.

As amostras de madeira para a determinação das propriedades físicas e mecânicas da madeira foram coletadas dos segundos melhores indivíduos de cada parcela (com base nos valores genéticos do DAP), sendo analisadas amostras de madeira das 120 famílias do teste de progênies, uma planta por parcela e cinco repetições, totalizando, assim, 600 árvores avaliadas.

\section{Coleta de amostras}

Nas árvores em pé, mensuraram-se os DAPs. Após o corte, foram mensuradas as alturas comerciais e seccionados os discos base, $25 \%, 50 \%, 75 \%$ e $100 \%$ de altura, para determinação da massa específica básica. Posteriormente, retiraram-se as primeiras e segundas toras para realização das demais análises físicas e mecânicas.

\section{Variáveis analisadas}

As variáveis avaliadas foram: massa específica básica e aparente, coeficientes de contração máxima volumétrica e linear tangencial, radial e longitudinal, anisotropia de contração e coeficientes de retratibilidade volumétrico, tangencial e radial, MOE e MOR, no ensaio de compressão pela grã, e tensão de cisalhamento.

\section{Massa específica básica}

$\mathrm{Na}$ determinação da massa específica básica (MEB), foram utilizados os discos retirados com aproximadamente $5 \mathrm{~cm}$ de espessura, conforme as alturas descritas anteriormente. Os discos foram imersos em recipiente com água até a completa saturação. Em seguida foi determinada a massa específica básica pelo método descrito na norma COPANT 461:1972.

\section{Retratibilidade}

Das segundas toras, provenientes da retirada dos discos nas posições base e $25 \%$ da altura comercial, foram retirados os corpos de prova de retratibilidade.

Para a confecção dos corpos de prova, foram destopados toretes com aproximadamente $50 \mathrm{~cm}$ e posteriormente retirados corpos de prova no sentido medula-casca, paralelamente à grã da madeira. Foram retirados quatro corpos de prova, denominados da seguinte forma: M1, M2 (retirados mais próximos à medula) e $\mathrm{C} 1, \mathrm{C} 2$ (retirados mais próximos à casca), a fim de que as médias resultantes abrangessem a maior parte possível do raio medula-casca.

As dimensões dos corpos de prova, bem como as análises, seguiram os critérios da norma COPANT 462:1972. Assim, foram determinados os coeficientes de contração máxima volumétrica e lineares (tangencial, radial e longitudinal), anisotropia de contração e coeficientes de retratibilidade volumétrico, tangencial e radial. Utilizaram-se as seguintes equações:

$$
\beta_{\text {(tang,rad,long })}=\left[\left(\mathrm{L}_{\mathrm{u}}-\mathrm{L}_{\mathrm{o}}\right) / \mathrm{L}_{\mathrm{u}}\right] * 100(\%)
$$

Contração linear máxima (1)

$\beta_{v o l}=\left[\left(\mathrm{V}_{\mathrm{u}}-\mathrm{V}_{\mathrm{o}}\right) / \mathrm{V}_{\mathrm{u}}\right] * 100(\%)$

$\mathrm{QR}=\left[\left(\mathrm{V}_{\text {clim }}-\mathrm{V}_{\mathrm{o}}\right) * \mathrm{P}_{\mathrm{o}}\right] /\left[\left(\mathrm{P}_{\text {clim }}-\mathrm{P}_{\mathrm{o}}\right) * \mathrm{I}_{\mathrm{o}}\right](\% / \%)$

$\mathrm{QR}_{\text {(tang,rad) }}=\left[\left(\mathrm{L}_{\text {clim }}-\mathrm{L}_{\mathrm{o}}\right) * \mathrm{P}_{\mathrm{o}}\right] /\left[\left(\mathrm{P}_{\text {clim }}-\mathrm{P}_{\mathrm{o}}\right) * \mathrm{~L}_{\mathrm{o}}\right](\% / \%)$

$\mathrm{AC}=\beta_{\text {tan }} / \beta_{\text {rad }}$
Contração linear máxima volumétrica (2)

Retração volumétrica (3)

Retração tangencial/radial (4)

Coeficiente de anisotropia (5) 
Em que: $\beta_{\text {(tang,rad,long })}=$ coeficiente de contração máxima linear (tangencial/radial/longitudinal);

$\beta_{v o l}=$ coeficiente de contração máxima volumétrica;

$\mathrm{QR}=$ coeficiente de retratibilidade;

$\mathrm{QR}_{\text {(tang,rad) }}=$ coeficiente de retratibilidade volumétrico (tangencial/radial);

$\mathrm{AC}=$ anisotropia de contração;

$\mathrm{L}_{\mathrm{u}}=$ dimensão no estado verde $(\mathrm{mm})$;

$\mathrm{L}_{\mathrm{o}}=$ dimensão após secagem em estufa a $103 \pm 2^{\circ} \mathrm{C}(\mathrm{mm})$;

$\mathrm{L}_{\text {clim }}=$ dimensão após condicionamento (mm);

$\mathrm{V}_{\mathrm{u}}=$ volume do corpo de prova no estado verde $\left(\mathrm{mm}^{3}\right)$;

$\mathrm{V}_{\mathrm{o}}=$ volume do corpo de prova seco em estufa a $103 \pm 2{ }^{\circ} \mathrm{C}\left(\mathrm{cm}^{3}\right)$;

$\mathrm{V}_{\text {clim }}=$ volume do corpo de prova após condicionamento $\left(\mathrm{mm}^{3}\right)$;

$\mathrm{P}_{\mathrm{o}}=$ massa do corpo de prova seca em estufa a $103 \pm 2{ }^{\circ} \mathrm{C}(\mathrm{g})$;

$\mathrm{P}_{\text {clim }}=$ massa do corpo de prova após climatização $(\mathrm{g})$.

Massa específica aparente

Na determinação da massa específica aparente, foram utilizados os mesmos corpos de prova da determinação da retratibilidade.

Os procedimentos para determinação da massa específica aparente foram efetuados conforme norma COPANT 461:1972, sendo tomadas as dimensões com auxílio de um paquímetro digital nos seguintes conteúdos de umidade: verde (acima do ponto de saturação das fibras), após acondicionamento dos corpos de prova em câmara aclimatizada (condições: UR $=65 \pm 5 \%$ e $\mathrm{T}=20 \pm 2{ }^{\circ} \mathrm{C}$ ) e a $0 \%$ de umidade (corpos de prova secos em estufa a $103 \pm 2{ }^{\circ} \mathrm{C}$ ). Para os cálculos, utilizou-se a equação 6:

$$
\mathrm{ME}_{\mathrm{ap}}=\mathrm{M}_{\mathrm{ap}} / \mathrm{V}_{\mathrm{ap}}
$$

Massa aparente (6)

Em que: $\mathrm{ME}_{\mathrm{ap}}=$ massa específica aparente $\left(\mathrm{g} / \mathrm{cm}^{3}\right)$;

$\mathrm{M}_{\mathrm{ap}}=$ massa do corpo de prova: verde, climatizado, $0 \%(\mathrm{~g})$;

$\mathrm{V}_{\mathrm{ap}}=$ volume do corpo de prova: verde, climatizado, $0 \%\left(\mathrm{~cm}^{3}\right)$.

Módulo de elasticidade à compressão

A confecção dos corpos de prova seguiu a mesma metodologia utilizada na retirada dos corpos de prova dos ensaios de retratibilidade, sendo retirados na sequência destes. Devido aos baixos diâmetros das toras, foram utilizados os corpos de prova com dimensões $25 \times 25 \times 100 \mathrm{~mm}$.

$\mathrm{O}$ ensaio foi realizado com a força atuando no sentido paralelo à grã da madeira. $\mathrm{O}$ módulo de elasticidade foi determinado segundo dados extraídos do diagrama carga-deformação, registrado pelo software Tesc® utilizado na máquina universal de ensaios EMIC e calculado segundo a norma EN789, conforme a seguinte equação:

$$
\mathrm{E}_{\mathrm{c}}=\left[\left(\mathrm{F}_{2}-\mathrm{F}_{1}\right) * 1_{1}\right] /\left[\left(\mathrm{u}_{2}-\mathrm{u}_{1}\right) * \mathrm{~A}\right]
$$

Módulo de elasticidade (7)

Em que: $\mathrm{E}_{\mathrm{c}}=$ módulo de elasticidade à compressão $\left(\mathrm{kgf} / \mathrm{cm}^{2}\right)$;

$\mathrm{F}_{2}=40 \%$ da força máxima $(\mathrm{kgf} /) ;$

$\mathrm{F}_{1}=10 \%$ da força máxima $(\mathrm{kgf} /)$;

$1_{1}=$ vão de deformação $(\mathrm{mm})$;

$\mathrm{u}_{1}=$ deformação correspondente a $10 \%$ da força máxima $(\mathrm{mm})$;

$\mathrm{u}_{2}=$ deformação correspondente a $40 \%$ da força máxima $(\mathrm{mm})$.

Sendo: $\quad A=$ área da seção $\left(\mathrm{mm}^{2}\right)$;

$\mathrm{A}=\mathrm{b} * \mathrm{~h}$;

$\mathrm{b}=$ largura do corpo de prova $(\mathrm{mm})$;

$\mathrm{h}=$ espessura do corpo de prova $(\mathrm{mm})$; 
Módulo de ruptura à compressão

O módulo de ruptura foi obtido através do mesmo diagrama de carga-deformação registrado pelo software Tesc® e calculado segundo a norma europeia EN789, segundo a equação 8:

$$
\mathrm{MOR}=\mathrm{F}_{\text {máx }} / \mathrm{A}
$$

Módulo de ruptura (8)

Em que: $\mathrm{MOR}=$ módulo de ruptura $\left(\mathrm{kgf} / \mathrm{cm}^{2}\right)$;

$\mathrm{F}_{\text {máx }}=$ força máxima $(\mathrm{kgf})$;

$\mathrm{A}=$ área da seção $\left(\mathrm{cm}^{2}\right)$;

Tensão de cisalhamento

Para a confecção dos corpos de prova de cisalhamento, foram seccionados primeiramente toretes com $30 \mathrm{~cm}$ e posteriormente retirados vigotes no mesmo comprimento, com medida de $1 \mathrm{~cm}$ em relação à seção transversal final do corpo de prova, os quais ficaram expostos em ambiente ventilado, para perda de umidade. Os vigotes foram orientados para serem retirados dos copos no sentido paralelo à grã da madeira. Posteriormente, foram confeccionados os corpos de prova e acondicionados em câmara aclimatizada até o momento do ensaio. Foram retirados de cada árvore avaliada dois corpos de provas, em raios opostos no sentido medula-casca. Com base em experiências prévias, verificou-se que duas amostras por árvore para análise de tensão de cisalhamento eram suficientes para atender os objetivos propostos neste trabalho para essa variável.

As dimensões dos corpos de prova e a realização dos ensaios seguiram os critérios da norma COPANT 463:1972. O ensaio foi realizado com a aplicação da força cortante atuando no sentido paralelo aos anéis de crescimento da madeira. A tensão de cisalhamento foi calculada com os resultados extraídos do software Tesc ${ }^{\circledR}$ utilizado na máquina universal de ensaios EMIC e conforme a seguinte equação:

$$
\mathrm{T}_{\text {cis }}=\mathrm{F}_{\text {máx }} / \mathrm{A}
$$

Tensão de cisalhamento máxima (9)

Em que: $\mathrm{T}_{\text {cis }}=$ tensão de cisalhamento máxima suportada $\left(\mathrm{kgf} / \mathrm{cm}^{2}\right)$;

$\mathrm{F}_{\text {máx }}=$ força máxima $(\mathrm{kgf})$;

$\mathrm{A}=$ área da superfície submetida ao cisalhamento $\left(\mathrm{cm}^{2}\right)$.

\section{Análise dos dados}

O agrupamento das famílias em função de suas características tecnológicas foi realizado por meio da técnica multivariada análise de componentes principais e análise de agrupamento (cluster).

A partir de uma matriz de médias dos 5 blocos das 120 famílias, foi realizada uma análise de componentes principais, os quais foram escolhidos de acordo com critério de Kaizer.

Para agrupamento das famílias, foi aplicada a técnica de agrupamento por meio do método $K$ means, utilizando-se a distância euclidiana média para cálculo das distâncias entre os grupos. Esse método permite a escolha prévia da quantidade de grupos a serem estabelecidos.

Para as análises, foi realizada, na matriz de médias, uma padronização por meio da equação $\mathrm{Xi}=\mathrm{Xi} / \sigma \mathrm{x}$, extraída de Cruz (2006). Essa padronização teve o objetivo de aproximar a distribuição das variáveis a uma normal. Assim, os dados das variáveis, mesmo existindo grandezas de valores diferenciados, puderam ser analisados dentro de uma mesma escala, facilitando análises posteriores.

As análises dos componentes principais e do agrupamento foram realizadas com auxílio do programa Statgraphics ${ }^{\circledR}$.

\section{RESULTADOS E DISCUSSÃO}

A análise de componentes principais permitiu destacar seis combinações lineares, que explicavam $82,51 \%$ da variação total. Dessa forma, a análise dos componentes principais possibilitou a obtenção de um pequeno número de combinações lineares das 17 variáveis, que explicam a maior parte da variabilidade dos dados. 
A partir da definição dos seis componentes principais, realizou-se a análise de agrupamento. Como parâmetro de agrupamento, indicaram-se seis seeds ou pontos de referência para os agrupamentos. Esses pontos de referência são as observações ou valores médios de famílias, em função do resultado dos componentes principais, ou seja, esses dados serviram como ponto de partida para estabelecer o número de agrupamentos desejados, conforme apresentado na tabela 1.

Tabela 1. Componentes, variáveis e pontos de referência para agrupamento.

Table 1. Components, variables and reference points for clustering.

\begin{tabular}{|c|c|}
\hline Componente & Variáveis e pontos de referência (seeds) \\
\hline & Referência: variável $\mathrm{ME}_{0 \%}$ \\
\hline 1 & Ponto de referência: família 11 (apresentou maior valor médio de $\mathrm{ME}_{0 \%}$ ) \\
\hline & Referência: variável AC \\
\hline 2 & Ponto de referência: família 64 (apresentou menor valor médio de AC) \\
\hline 3 & $\begin{array}{l}\text { Referência: relação entre as variáveis DAP e } \mathrm{H}_{\text {com }} \\
\text { Ponto de referência: família } 38 \text { (apresentou maior relação DAP/ } \mathrm{H}_{\text {com }} \text { ) }\end{array}$ \\
\hline 4 & $\begin{array}{l}\text { Referência: variável } \beta_{\text {long }} \\
\text { Ponto de referência: família } 111 \text { (apresentou menor valor médio de } \beta_{\text {long }} \text { ) }\end{array}$ \\
\hline 5 & $\begin{array}{l}\text { Referência: variável MOR } \\
\text { Ponto de referência: família } 20 \text { (apresentou maior valor médio de MOR) }\end{array}$ \\
\hline 6 & $\begin{array}{l}\text { Referência: variável } \mathrm{T}_{\text {cis }} \\
\text { Ponto de referência: família } 82 \text { (apresentou maior valor médio de } \mathrm{T}_{\text {cis }} \text { ) }\end{array}$ \\
\hline
\end{tabular}

Nota: MOR: módulo de ruptura $\left(\mathrm{kgf} / \mathrm{cm}^{2}\right) ; \mathrm{T}_{\mathrm{CIS}}$ : tensão de cisalhamento $\left(\mathrm{kgf} / \mathrm{cm}^{2}\right) ; \beta_{\text {long: }}$ : coeficiente de contração máxima linear volumétrica; AC: coeficiente de anisotropia $\left(\beta_{\text {tan }} / \beta_{r a d}\right) ; \mathrm{ME}_{0 \%}$ : massa específica seca a $0 \%\left(\mathrm{~kg} / \mathrm{m}^{3}\right) ; \mathrm{H}_{\mathrm{com}}$ : altura comercial $(\mathrm{m}) ; \mathrm{DAP}$ : diâmetro à altura do peito $(\mathrm{cm})$.

Na tabela 2 são apresentadas as médias das variáveis avaliadas nos grupos, determinadas pela análise de agrupamento dos seis clusters.

Tabela 2. Médias das variáveis avaliadas nos grupos, determinadas pela análise de agrupamento. Table 2. Variable averages in groups determined by cluster analysis.

\begin{tabular}{lcccccc}
\hline Variável & $\mathbf{1}$ & $\mathbf{2}$ & $\mathbf{3}$ Clusters & $\mathbf{4}$ & $\mathbf{5}$ & $\mathbf{6}$ \\
& $\mathbf{n = 1 2}$ & $\mathbf{n = 2 0}$ & $\mathbf{n = 3 1}$ & $\mathbf{n = 2 5}$ & $\mathbf{n = 1 3}$ & $\mathbf{n = 1 9}$ \\
\hline $\mathrm{H}_{\text {com }}$ & 23,88 & 23,5 & 23,27 & 23,48 & 22,98 & 23,1 \\
$\mathrm{DAP}$ & 21,54 & 20,88 & 20,75 & 20,68 & 20,19 & 20,36 \\
$\mathrm{~T}_{\mathrm{CIS}}$ & 90,35 & 77,93 & 86,86 & 91,17 & 101,2 & 82,71 \\
$\mathrm{MOR}$ & 248,28 & 232,09 & 227,55 & 237,07 & 241,53 & 244,5 \\
$\mathrm{MOE}$ & 12344,29 & 11647,01 & 11268,04 & 11956,27 & 11922,2 & 12308,09 \\
$\mathrm{MEB}$ & 402 & 379,24 & 384,77 & 390,35 & 393,3 & 384,19 \\
$\mathrm{ME}_{\text {verde }}$ & 0,6 & 0,57 & 0,57 & 0,59 & 0,61 & 0,59 \\
$\mathrm{ME}_{\text {clim }}$ & 0,44 & 0,41 & 0,41 & 0,42 & 0,42 & 0,42 \\
$\mathrm{ME}_{0 \%}$ & 0,41 & 0,37 & 0,37 & 0,38 & 0,39 & 0,39 \\
$\beta_{\text {vol }}$ & 8,88 & 7,96 & 8,11 & 8,46 & 8,29 & 8,58 \\
$\beta_{\text {tang }}$ & 6,44 & 5,74 & 5,83 & 6,14 & 5,96 & 6,26 \\
$\beta_{\text {rad }}$ & 3,2 & 2,82 & 2,9 & 3,02 & 3,01 & 3,03 \\
$\beta_{\text {long }}$ & 0,24 & 0,27 & 0,29 & 0,26 & 0,24 & 0,22 \\
$\mathrm{AC}$ & 2,06 & 2,1 & 2,09 & 2,1 & 2,04 & 2,13 \\
$Q R_{\text {vol }}$ & 0,38 & 0,34 & 0,34 & 0,35 & 0,35 & 0,36 \\
$Q R_{\text {tang }}$ & 0,35 & 0,32 & 0,32 & 0,34 & 0,32 & 0,35 \\
$Q R_{\text {rad }}$ & 0,13 & 0,11 & 0,11 & 0,12 & 0,12 & 0,12
\end{tabular}

Nota: MEB: média da família $\left(\mathrm{kg} / \mathrm{m}^{3}\right)$; MOR: módulo de ruptura $\left(\mathrm{kgf} / \mathrm{cm}^{2}\right) ;$ MOE módulo de elasticidade $\left(\mathrm{kgf} / \mathrm{cm}^{2}\right) ; \mathrm{T}_{\mathrm{CIS}}$ : tensão de cisalhamento $\left(\mathrm{kgf} / \mathrm{cm}^{2}\right) ; \beta_{\text {vol }} \beta_{\text {tang, }} \beta_{\text {rad, }} \beta_{\text {long: }}$ : coeficientes de contração máxima linear volumétrica, tangencial radial e longitudinal (\%); AC: coeficiente de anisotropia $\left(\beta_{\text {tan }} / \beta_{\text {rad }}\right) ; Q R_{\text {vol }}, Q R_{\text {tang }}, Q R_{\text {rad: coeficientes de contração volumétrico, tangencial e radial }}$ $(\% / \%) ; \mathrm{ME}_{\text {verde }}$ : massa específica verde $\left(\mathrm{kg} / \mathrm{m}^{3}\right) ; \mathrm{ME}_{\text {clim: }}$ massa específica climatizada $\left(\mathrm{kg} / \mathrm{m}^{3}\right) ; \mathrm{ME}_{0 \%}$ : massa específica seca a $0 \%$ $\left(\mathrm{kg} / \mathrm{m}^{3}\right) ; \mathrm{H}_{\text {com: }}$ altura comercial $(\mathrm{m}) ;$ DAP: diâmetro à altura do peito $(\mathrm{cm})$. 
Observando-se a tabela 2, as seguintes informações podem ser extraídas dos clusters formados:

- Cluster 1: as maiores médias encontradas em relação a esse grupo foram para as variáveis $\mathrm{H}_{\text {com }}$, DAP, $\mathrm{MOR}, \mathrm{MOE}, \mathrm{MEB}, \mathrm{ME}_{\mathrm{clim}}$ e $\mathrm{ME}_{0 \%}$, que respondem melhor à produtividade e à resistência, podendo-se indicar as famílias pertencentes a esse grupo para fins mais nobres, como estruturas em madeira, móveis e ainda para a seleção baseada em produtividade, por exemplo, para a indústria de celulose e papel.

- Cluster 2: as maiores médias encontradas em relação a esse grupo foram para as variáveis de resposta da retratibilidade da madeira $\left(\beta_{\text {vol }}, \beta_{\text {tang }}, \beta_{\text {rad }}, Q R_{\text {vol }}, Q R_{\text {tang }}\right.$ e $\left.Q R_{\text {rad }}\right)$, podendo ser indicadas para aplicações que requeiram maior estabilidade do material, como pisos, esquadrilhas e encaixes.

Ainda podem ser destacados o cluster 5, para melhor coeficiente de anisotropia e maior massa específica aparente verde (>28\% de umidade), e o cluster 6 , para maior $\beta_{\text {long }}$, indicando o menor desempenho de lenho juvenil, por esta variável ser influenciada pelo comportamento mais instável desse tipo de madeira.

A tabela 3 apresenta as famílias alocadas em cada cluster com suas respectivas finalidades.

Tabela 3. Famílias designadas por cluster.

Table 3. Families referred to as cluster.

\begin{tabular}{lcc}
\hline Clusters & Famílias & Finalidade \\
\hline 1 & $5,8,11,16,24,35,75,79,83,89,108,115$ & Produtividade / Resistência \\
2 & $12,15,17,19,21,33,40,53,56,58,60,74$, & Estabilidade dimensional \\
& $81,94,98,101,107,112,113$ & \\
3 & $3,4,6,22,26,29,36,38,43,47,48,51,63$, & ---------- \\
& $67,69,73,80,88,92,93,95,96,97,99,104$ & \\
4 & $106,117,118,120$ & - \\
& $1,7,14,23,27,28,37,41,42,45,52,55,59$, & Menor anisotropia e maior \\
5 & $64,68,70,72,84,85,86,91,103,110$ & massa específica verde \\
& $10,18,25,32,44,46,50,54,65,82,90,100$ & Menor desempenho para \\
6 & $2,9,13,20,30,31,34,39,49,61,66,71,78$, & madeira de lenho juvenil \\
\hline
\end{tabular}

No cluster 1, pode-se observar que as 12 famílias contidas nesse grupo foram as que reuniram mais variáveis para a seleção, em vista das suas características tecnológicas. Contudo, com relação às variáveis DAP e $\mathrm{H}_{\text {com }}$, a correlação obtida foi de 0,78 . Nesse caso, deve-se basear a seleção no melhoramento em apenas uma das duas características, pelo fato de elas apresentarem tendência linear e positiva. Ainda no cluster 1, as variáveis MOE e MOR apresentaram também uma correlação moderada e positiva, podendo-se selecionar apenas para uma das duas, recomendando-se selecionar para a variável MOR, uma vez que apresentou correlações moderadas e positivas com as outras duas variáveis que destacaram o grupo, $\mathrm{ME}_{\text {clim }} \mathrm{e}$ $\mathrm{ME}_{0 \%}$. Caso a opção seja indicar no grupo apenas uma característica a ser selecionada, a variável $\mathrm{ME}_{0 \%}$ (massa específica aparente a $0 \%$ de umidade) seria a mais recomendável, por ter apresentado maiores correlações com as demais e por ser de mais fácil determinação. Principalmente no caso de seleções precoces, em que a massa específica seca é determinada a partir de pequenas amostras verdes (galhos), para se calcular o potencial de matéria lenhosa para aquele indivíduo (árvore) futuramente.

Para o cluster 2 , as variáveis $\beta_{\text {vol }}, \beta_{\text {tang }}, \beta_{\text {rad }}, Q R_{\text {vol }}, Q R_{\text {tang }}$ e $Q R_{\text {rad }}$, que destacaram esse agrupamento, apresentaram, no estudo de suas correlações entre elas, tendências fortes e positivas conceitualmente esperadas. Assim, recomenda-se selecionar para apenas uma dessas características, sendo a variável $\beta_{v o l}$ a mais recomendável, por ter apresentado, com a massa específica aparente, correlações moderadas, positivas e mais significativas que as demais variáveis desse grupo.

O cluster 3 destacou-se como o de menor rigidez e resistência, uma vez que as variáveis MOE e MOR foram as menores em relação aos demais clusters.

O cluster 4 não teve destaque para nenhuma característica em especial, ficando entre as médias dos demais clusters.

Chama-se a atenção às famílias constantes no cluster 5 , por conterem os maiores valores para massa específica básica, mas não sendo recomendado tal grupo, pelo fato de conter mais umidade, cuja 
presença acarreta mais peso à madeira, significando mais custos no transporte, uma vez que se estará transportando mais água do que matéria seca.

As famílias do cluster 6 devem ser evitadas em uma seleção em que se deseje maior estabilidade da madeira, pois a variável $\beta_{\text {long }}$ indica, conforme já discutido no estudo das correlações dessa variável, uma menor estabilidade do material, por representar maior contração dos sentidos tangenciais, o que causa mais torções do lenho juvenil, de maneira a causar maior expansão nas dimensões do sentido longitudinal.

\section{CONCLUSÕES}

- As 120 famílias de meio-irmãos de Pinus taeda L. se agruparam por similaridade em 6 grupos, com base em um conjunto de características físicas e mecânicas.

- Os seis grupos formados reúnem distintas características físicas e mecânicas da madeira relacionadas à produção de madeira para diferentes finalidades.

- O grupo de famílias que compõem o cluster 1, constituído de 12 famílias, é indicado para finalidades que obtenham maior valor agregado, como estruturas em madeira, móveis, celulose e papel. O grupo de famílias do cluster 2 (19 famílias) é indicado para aplicações que requeiram maior estabilidade do material, como pisos, esquadrias e encaixes. As famílias alocadas no cluster 5 (12 famílias) apresentaram melhor coeficiente de anisotropia e maior massa específica aparente verde. As 18 famílias pertencentes ao cluster 6 apresentaram menor desempenho em comparação às outras famílias, quando utilizada madeira de lenho juvenil.

\section{AGRADECIMENTOS}

À Universidade Federal do Paraná (UFPR), à empresa Battistella Florestal e à Financiadora de Estudos e Projetos (FINEP).

\section{REFERÊNCIAS}

ASSOCIAÇÃO BRASILEIRA DE PRODUTORES DE FLORESTAS PLANTADAS (ABRAF). Anuário estatístico da ABRAF 2011 ano base 2010/ ABRAF. Brasília: 2011. 130 p.

ASSIS, T. F. Estratégias de melhoramento para obtenção de madeira de qualidade para laminação e serraria. In: SEMINÁRIO DE MADEIRA DE EUCALIPTO: TENDÊNCIAS E USOS. 2001, Curitiba. Anais... Curitiba, 2001.p. 1 - 18.

CAIXETA, R. P.; TRUGILHO, P. F.; ROSADO, S. C. S.; LIMA J. T. Propriedades e classificação da madeira aplicadas à seleção de genótipos de Eucalyptus. Revista Árvore. v. 27, n. 1, p. 43 - 51, 2003.

CARLE, J.; HOLMGREN, P. Wood from planted forest: a global outlook 2005 - 2030. Forest Products Journal, v. 58, n. 12, p. 6 - 13, 2008.

CRUZ, C. D. Programa Genes: análise multivariada e simulação. Viçosa: Editora UFV, 2006. 175 p.

FOELKEL, E. Poda, desrama e desbaste das árvores de Pinus para melhor qualidade da madeira. Disponível em: 〈http://www.celso-foelkel.com.br/pinus_14.html\#quatorze〉. Acesso em 03/04/2008.

LOPES, M. C. Agrupamento de árvores matrizes de Eucalyptus grandis em função das variáveis dendrométricas e das características tecnológicas da madeira. $111 \mathrm{f}$. Dissertação (Mestrado em Engenharia Florestal) - Universidade Federal de Santa Maria. Santa Maria, 2003.

RODRIGUES, E. A. C.; ROSADO, S. C. S.; TRUGILHO, F. P.; SANTOS, A. M. Seleção de clones de Eucalyptus para as propriedades físicas da madeira avaliadas em árvores no campo. Revista Cerne, v. 14, n. 2, p. $147-152,2008$.

SOCIEDADE BRASILEIRA DE SILVICULTURA (SBS). Fatos e números do Brasil Florestal. São Paulo: SBS, 2008. 93 p.

Florestas do Brasil em resumo. Brasília: SBF, 2010. 152 p. 